\title{
Design Method Research on Autopilot of Rolling Missile
}

\author{
Yuan Bo \\ School of Astronautics \\ Northwestern Polytechnical University \\ Xi'an, China \\ e-mail: ybstrong@126.com
}

\author{
Yang Jun \\ School of Astronautics \\ Northwestern Polytechnical University \\ Xi'an, China \\ e-mail: yangjunlsyx@126.com
}

\begin{abstract}
The theory on autopilot design of non-rolling missile has been well developed, but the perfect theory on autopilot design of rolling missile has not come into being. Aiming at this problem, the coherence research on autopilot is carried out based on the analysis of scope and frequency area. And the primary coherence condition is given. With the research work, it is expected that the answer can be gotten about with which condition controller design of rolling missile can be translated into controller design of non-rolling missile. Finally, the actuator delay problem of rolling missile is researched, too.
\end{abstract}

Keywords- Autopilot design; rolling missile; coherence research; actuator delay

\section{INTRODUCTION}

Nowadays, a complete design method on autopilot design of rolling missile has not come into being, and the empirical method and method of the trial and error is widely used in engineering.

Considering the design of autopilot of non-rolling missile has perfect theory, a method is to design autopilot of rolling missile with non-rolling assumption firstly, and then to eliminate the effect of rotation with compensation algorithm design.

The premise of this method is that the control performance of missile regardless of rotation or non-rotation is same or basically same. However, not all of the autopilot structures can satisfy the premise. Aiming at this problem, the coherence problem on autopilot is carried out in this paper, it is expected with research work that the answer can be gotten about with which condition controller design of rolling missile can be translated into controller design of non-rolling missile.

\section{COHERENCE CONDITION ANALYSES}

To research the coherence problem, the quasi body coordinate system is given firstly, and the transformation between the quasi body coordinate system $O x_{1}^{\prime} y_{1}^{\prime} z_{1}^{\prime}$ and the body coordinate system $O x_{1} y_{1} z_{1}$ is given as follow:

$$
\begin{gathered}
T_{1^{\prime} \rightarrow 1}=\left[\begin{array}{ccc}
1 & 0 & 0 \\
0 & \cos \tilde{\gamma} & \sin \tilde{\gamma} \\
0 & -\sin \tilde{\gamma} & \cos \tilde{\gamma}
\end{array}\right] \\
\tilde{\gamma}=\bar{\omega}_{x} \cdot t
\end{gathered}
$$

Where, $\tilde{\gamma}$ is the included angle between quasi body coordinate plane $x_{1}^{\prime} O_{1}^{\prime} y_{1}^{\prime}$ and body coordinate plane $x_{1} O_{1} y_{1}$, $\bar{\omega}_{x}$ is rolling rate of missile, $t$ is length of rolling time.

Given a step command along direction of axis $O_{1}^{\prime} y_{1}^{\prime}$, defined as $u=n_{c}$. With condition of non-rolling, the response of axis $O_{1} y_{1}$ is described as follows:

$$
y_{1}=\left.A(\omega) \cdot n_{c} \cdot \sin \left(\omega t+\frac{\pi}{2}+\varphi(\omega)\right)\right|_{\omega=0}
$$

The closed loop transfer function of typical autopilot is no zero pole, so there is

$$
\left.\varphi(\omega)\right|_{\omega=0}=0
$$

With this,

$$
y_{1}=\left.A(\omega) \cdot n_{c} \cdot \sin \left(\omega t+\frac{\pi}{2}\right)\right|_{\omega=0}=A(0) \cdot u
$$

With condition of rolling, the command of along direction of axis $O_{1} y_{1}$ is

$$
u_{1}=n_{c} \cdot \cos \omega_{0} t=n_{c} \cdot \sin \left(\omega_{0} t+\frac{\pi}{2}\right)
$$

The response of axis $O_{1} y_{1}$ is described as follows:

$$
y_{1}=\left.A(\omega) \cdot n_{c} \cdot \sin \left(\omega t+\frac{\pi}{2}+\varphi(\omega)\right)\right|_{\omega=\omega_{0}}
$$

In order to ensure coherence of control performance between rolling missile autopilot and non-rolling missile autopilot, conditions as follows should be satisfied:

$$
\begin{aligned}
& A(0)=A\left(\omega_{0}\right) \\
& \varphi(0)=0=\varphi\left(\omega_{0}\right)
\end{aligned}
$$

The approximate condition of coherence is given as follows:

$$
\begin{aligned}
& \left|A(0)-A\left(\omega_{0}\right)\right| \leq \varepsilon_{A} \\
& \left|\varphi(0)-\varphi\left(\omega_{0}\right)\right| \leq \varepsilon_{\varphi}
\end{aligned}
$$

Where, $\varepsilon_{A}$ and $\varepsilon_{\varphi}$ are small non-negative value, and the approximate condition of coherence becomes strict condition of coherence with zero value of $\varepsilon_{A}$ and $\varepsilon_{\varphi}$. 


\section{COHERENCE ANALYSES OF SEVERAL AUTOPILOT STRUCTURES}

According to the conclusion of the above theoretical analysis, coherence analyses of several autopilot structures will be researched, including open-loop autopilot, angular rate feedback autopilot and angular rate feedback+ overload feedback autopilot.

\section{A. Coherence analyses of open-loop autopilot}

Open-loop autopilot structure diagram with actuator characteristics ignored is shown in figure 1.

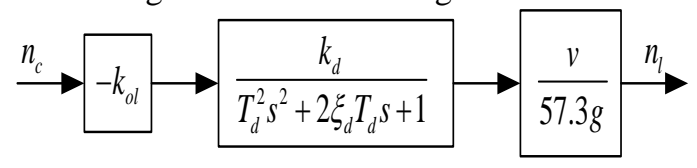

Figure 1. Open-loop autopilot structure diagram.

Transfer function of open-loop autopilot is given as follows:

$$
\phi(s)=-k_{O L} \cdot \frac{v}{57.3 g} \cdot \frac{k_{d}}{T_{d}^{2} s^{2}+2 \xi_{d} T_{d} s+1}
$$

Considering the desired steady-state transfer gain is 1 , there is,

$$
k_{O L}=-\frac{57.3 g}{v \cdot k_{d}}
$$

Then,

$$
\phi(s)=\frac{1}{T_{d}^{2} s^{2}+2 \xi_{d} T_{d} s+1}
$$

The amplitude frequency characteristic and phase frequency characteristic formula is as follows:

$$
\begin{gathered}
A(\omega)=\frac{1}{\sqrt{\left(1-T_{d}^{2} \omega^{2}\right)^{2}+\left(2 \xi_{d} T_{d} \omega\right)^{2}}} \\
\varphi(\omega)=-\arctan \frac{2 \xi_{d} T_{d} \omega}{1-T_{d}^{2} \omega^{2}} \\
\text { If } T_{d} \omega_{0} \square 1, \text { then } \quad A(0)=1 \\
A\left(\omega_{0}\right) \approx \frac{1}{T_{d}^{2} \omega_{0}^{2}} \\
\varphi(0)=0 \\
\varphi\left(\omega_{0}\right)=\arctan \frac{2 \xi_{d}}{T_{d} \omega_{0}}-180^{\circ}
\end{gathered}
$$

It can be seen from the above analysis that the approximate condition of coherence is difficult to be satisfied with $T_{d} \omega_{0} \square 1$ and is very likely to be satisfied with $T_{d} \omega_{0} \square 1$. Therefore, its premise of coherence condition of open-loop autopilot is $T_{d} \omega_{0} \square 1$.

\section{B. Coherence analyses of angular rate feedback autopilot}

Angular rate feedback autopilot structure diagram with actuator and rate gyroscope characteristics ignored is shown in figure 2 .

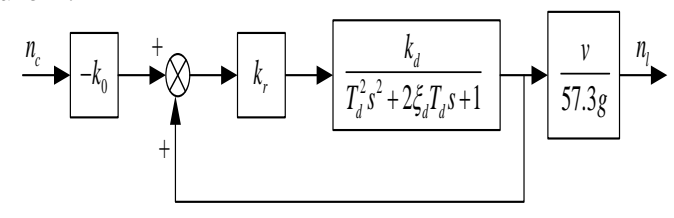

Figure 2. Angular rate feedback autopilot structure diagram.

Transfer function of angular rate feedback autopilot is given as follows:

$$
\phi(s)=-k_{0} \cdot \frac{v}{57.3 g} \cdot \frac{k_{d}^{\prime}}{T_{d}^{\prime 2} s^{2}+2 \xi_{d}^{\prime} T_{d}^{\prime} s+1}
$$

Where,

$$
\begin{aligned}
k_{d}^{\prime} & =\frac{k_{d}}{1-k_{d} k_{r}} \\
T_{d}^{\prime} & =\frac{T_{d}}{\sqrt{1-k_{d} k_{r}}} \\
\xi_{d}^{\prime} & =\frac{\xi_{d}}{\sqrt{1-k_{d} k_{r}}}
\end{aligned}
$$

Considering the desired steady-state transfer gain is 1 , there is,

$$
k_{0}=-\frac{57.3 g}{v \cdot k_{d}^{\prime}}
$$

Then,

$$
\phi(s)=\frac{1}{T_{d}^{\prime 2} s^{2}+2 \xi_{d}^{\prime} T_{d}^{\prime} s+1}
$$

The amplitude frequency characteristic and phase frequency characteristic formula is as follows:

$$
\begin{gathered}
A(\omega)=\frac{1}{\sqrt{\left(1-T_{d}^{\prime 2} \omega^{2}\right)+\left(2 \xi_{d}^{\prime} T_{d}^{\prime} \omega\right)^{2}}} \\
\varphi(\omega)=-\arctan \frac{2 \xi_{d}^{\prime} T_{d}^{\prime} \omega}{1-T_{d}^{\prime 2} \omega^{2}} \\
\text { If } T_{d} \omega_{0} \square 1, \text { then, } \\
A(0)=1 \\
A\left(\omega_{0}\right) \approx 1 \\
\varphi(0)=0 \\
\varphi\left(\omega_{0}\right)=\arctan 2 \xi_{d}^{\prime} T_{d}^{\prime} \omega_{0} \\
\text { If } T_{d} \omega_{0} \square 1, \text { then } \\
A(0)=1 \\
A\left(\omega_{0}\right) \approx \frac{1}{T_{d}^{\prime 2} \omega_{0}^{2}}
\end{gathered}
$$




$$
\begin{gathered}
\varphi(0)=0 \\
\varphi\left(\omega_{0}\right)=\arctan \frac{2 \xi_{d}^{\prime}}{T_{d}^{\prime} \omega_{0}}-180^{\circ}
\end{gathered}
$$

Similar with coherence condition of open-loop autopilot, its premise of coherence condition of angular rate feedback autopilot is $T_{d} \omega_{0} \square 1$. With the consideration of $T_{d}^{\prime}<T_{d}$, angular rate feedback autopilot is easier to meet the premise than open-loop autopilot.

\section{Coherence analyses of angular rate feedback+ overload feedback autopilot}

1) Proportional type overload feedback autopilot

Transfer function of angular rate feedback loop is approximate to steady-state gain $k_{\omega}$. And proportional type overload feedback autopilot is shown in figure 3 with actuator and rate gyroscope characteristics ignored.

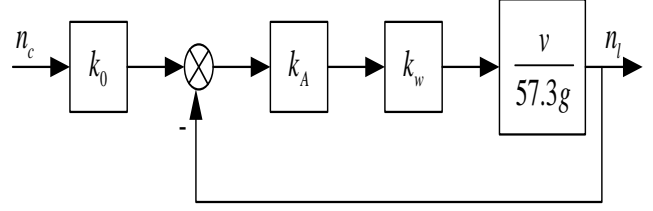

Figure 3. Angular rate feedback autopilot structure diagram.

Transfer function of proportional type overload feedback autopilot is given as follows:

$$
\phi(s)=\frac{k_{0} \cdot k_{A} k_{\omega} v / 57.3 g}{1+k_{A} k_{\omega} v / 57.3 g}
$$

Considering the desired steady-state transfer gain is 1 , there is,

$$
k_{0}=\frac{1+k_{A} k_{\omega} v / 57.3 g}{k_{A} k_{\omega} v / 57.3 g}
$$

Then,

$$
\phi(s)=1
$$

It can be seen from the above analysis that proportional type overload feedback autopilot is satisfied with coherence condition.

2) Integral type overload feedback autopilot

Transfer function of angular rate feedback loop is approximate to steady-state gain $k_{\omega}$. Integral type overload feedback autopilot is shown in figure 4 with actuator and rate gyroscope characteristics ignored.

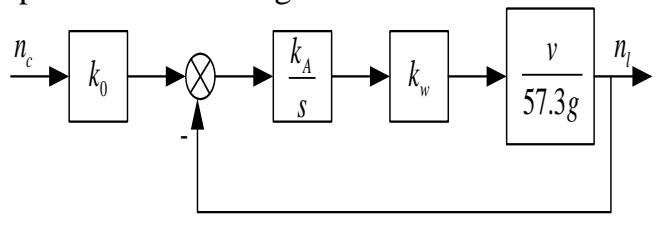

Figure 4. Integral type overload feedback autopilot structure diagram.

Transfer function of integral type overload feedback autopilot is given as follows:

$$
\phi(s)=\frac{k_{0}}{T^{\prime} s+1}
$$

Where,

$$
T^{\prime}=\frac{57.3 g}{k_{A} k_{w} v}
$$

Considering the desired steady-state transfer gain is 1 , there is,

$$
k_{0}=1
$$

Then,

$$
\phi(s)=\frac{1}{T^{\prime} s+1}
$$

The amplitude frequency characteristic and phase frequency characteristic formula is as follows:

$$
\begin{aligned}
A(\omega) & =\frac{1}{\sqrt{1+T^{\prime 2} \omega^{2}}} \\
\varphi(\omega) & =-\arctan T^{\prime} \omega
\end{aligned}
$$

Where,

$$
T^{\prime}=\frac{57.3 g}{k_{A} k_{w} v}
$$

Then,

$$
\begin{gathered}
A(0)=1 \\
A\left(\omega_{0}\right) \approx \frac{1}{T^{\prime} \omega_{0}} \\
\varphi(0)=0 \\
\varphi\left(\omega_{0}\right) \approx-\left(90^{\circ}-\arctan \frac{1}{T^{\prime} \omega_{0}}\right)
\end{gathered}
$$

According to above formulas, there is $\varphi\left(\omega_{0}\right)<-45^{\circ}$. Therefore integral type overload feedback autopilot is not satisfied with coherence condition.

\section{Compensation algorithm of phase delay of rolling missile actuator}

The frequency band of rudder deflection angle of nonrolling missile is low, so phase delay of rolling missile actuator can be ignored. And phase delay of rolling missile actuator cannot be ignored because of the signal of rudder deflection angle is modulated by rotation. So it is necessary to research Compensation algorithm of phase delay of rolling missile actuator.

Lead corrector as follows can be designed to compensate the phase delay.

$$
a G_{c}(s)=\frac{1+a T s}{1+T s}
$$

Firstly, corresponding phase delay $\phi$ of actuator at rotation frequency $\bar{\omega}_{x}$ can be obtained with phase frequency curve of actuator. And parameter $a$ and T can be calculated with formula as follows. 


$$
\begin{gathered}
a=\frac{1+\sin \phi}{1-\sin \phi} \\
T=\frac{1}{\bar{\omega}_{x} \sqrt{a}}
\end{gathered}
$$

Thus, actuator compensation algorithm is realized.

\section{CONCLUSION}

With the research work in this paper, it can be drawn that not all kinds of autopilot structures can be designed with design method of non-rolling missile, and for those autopilot structures which can be designed with design method of nonrolling missile, some condition should be satisfied.

\section{REFERENCES}

[1] Garnell P. , Guided Weapon Control System. Beijing: Beijing Institute of Technology, 2003.

[2] Zhang Xin-guo, Zhang Ru-lin, Chen Zong-ji, "Design Methods for Flight Control Systems," Aeronautical Science and Technology, Jan. 1997, pp. 17-31.

[3] FAN Jun-fang, LIN De-fu, QI Zai-kang, ZHANG Hong, "Design and analysis of a two-loop autopilot", Systems Engineering and Electronics, Vol. 30, Dec. 2008, pp. 2447-2450.

[4] Nesline F.W., Zarchan P., "A classical look at modern control for missile autopilot design", AIAA Guidance and Control Conference, Aug. 1979, Paper No. 82-1512, pp. 90-104.

[5] Jiang Zhong-ming, "Designing and Simulating Control System for a Self-rotary Missile of The Air Defense Missile/Gun Weapon System," Master. dissertation, NWPU Univ., Xi'an, China, 2005. 